\title{
PRODUCTION D'ÉLÉMENTS ORBITAUX DE COMÈTES
}

\section{SUR PC}

\author{
P. ROCHER ET C. CAVELIER \\ Bureau des longitudes \\ 77, avenue Denfert-Rochereau \\ 75014, Paris FRANCE
}

\section{Introduction}

La puissance de calcul des ordinateurs PC actuels permet leur utilisation pour des travaux qui étaient, jusqu'à ces dernières années, effectués exclusivement sur de gros ordinateurs. Le mode interactif utilisé pour la gestion et la saisie des données, facilement mis en œuvre sur les PC, permet un gain de temps important par rapport au travail classique en traitement par lot (batch). Nous présentons ici un logiciel PC, permettant le calcul et l'amélioration des éléments orbitaux des comètes. Une bonne connaissance de ces éléments est indispensable pour le calcul des éphémérides, notamment pour la redécouverte des comètes périodiques.

\section{Description du logiciel}

Le programme est écrit en Pascal 7.0 (Borland Pascal ${ }^{\circledR}$ ) et utilise un logiciel de gestion d'écrans et de menus déroulants Hyper-Screen 5.5 (PC Soft $\left.{ }^{\circledR}\right)$. Il fonctionne sur des ordinateurs PC (486 recommandé) ayant au moins 4 Mo de RAM. Il occupe environ 15 Mo sur le disque dur. La totalité du programme fonctionne en mode interactif, en utilisant des menus déroulants et des écrans de saisies et d'affichages, le tout étant géré à l'aide de la souris.

Ce programme permet donc le calcul et l'amélioration des éléments orbitaux des comètes à l'aide des observations. On peut distinguer trois phases importantes : la gestion des différentes bases de données liées au problème, le calcul proprement dit, et la sortie et la publication des résultats. 


\section{Gestion des bases de données}

Ce programme utilise de nombreuses bases de données.

\subsection{POUR LES OBSERVATIONS}

On dispose de trois bases de données, une pour les observations des comètes périodiques (environ 28000 observations), une pour les comètes non périodiques (environ 18000 observations) et une pour les lieux d'observations (environ 530 sites). Ces différentes bases sont mises à jour régulièrement à l'aide des données publiées dans les Minor Planet Circulars. Le programme permet la gestion de ces bases, notamment le tri et la mise à jour des fichiers d'observations.

\subsection{POUR LES ÉLÉMENTS ORBITAUX}

Les éléments orbitaux sont sauvegardés sous deux formes distinctes : soit d'une part des fichiers ASCII contenant les éléments sous la forme de conditions initiales (vecteurs position et vitesse), soit d'autre part des bases de données " éléments cométaires 》 dans des fichiers séquentiels indexés utilisant la méthode des arbres $B$. La première forme est utilisée uniquement par ce programme alors que la seconde est utilisée par d'autres programmes, notamment pour le calcul et l'édition des éphémérides de comètes.

\subsection{POUR LES ÉPHÉMÉRIDES DES PLANĖTES PERTURBATRICES}

Pour le calcul des orbites, on tient compte de l'ensemble des perturbations planétaires et lunaire. Les éphémérides de ces corps sont issues des théories élaborées au Bureau des longitudes, VSOP82 (P. Bretagnon 1982) et TOP82 (J.L. Simon, 1983) pour les planètes et ELP-2000/82 (M. Chapront-Touzé et J. Chapront, 1983) pour la Lune. Les positions sont calculées à partir de représentations en séries de polynômes de Tchebycheff des éphémérides issues de ces théories (G. Francou et al., 1983). Les éphémérides que nous utilisons couvrent un intervalle de temps de deux siècles ayant de 1850 à 2050 .

\section{Calcul et méthodes}

\section{1. ÉQUATIONS DU MOUVEMENT}

Les équations du mouvement sont écrites dans le repère cartésien équatorial héliocentrique J2000. Pour le calcul de la trajectoire, on tient compte de l'ensemble des perturbations planétaires et lunaire, ainsi que des effets des 
forces de type non gravitationnel (forces dues au dégazage du noyau au voisinage du Soleil) lorsque cela est nécessaire.

\subsection{INTÉGRATION NUMÉRIQUE ET COMPARAISON AUX OBSERVATIONS}

Les observations sont des coordonnées équatoriales astrographiques topocentriques J2000. Les instants d'observations sont en Temps universel. On calcule pour chaque observation la position et la vitesse de la comète à l'aide d'une intégration numérique. On utilise pour cela la méthode d'intégration de Gragg-Bulirsh-Stoer (R. Bulirsh. et J. Stœr, 1980), il s'agit d'une méthode de type multi prédicteurs-correcteurs, la prédiction se fait par la méthode du point milieu et la correction par extrapolation en fractions rationnelles. En plus de la position et de la vitesse de la comète, on intègre également un système d'équations différentielles permettant de calculer en chaque point d'observation les dérivées des variables par rapport aux paramètres de l'intégration (conditions initiales et coefficients des forces non gravitationnelles). Ces dérivées sont utilisées par la suite pour l'amélioration de l'orbite.

\subsection{AMÉLIORATION DES PARAMĖTRES}

Selon que l'on utilise où non des forces non gravitationnelles, le nombre de paramètres à ajuster peut être de 6,8 ou 9 . On ajuste alors les positions et les vitesses initiales, ainsi que les coefficients des forces non gravitationnelles. Cet ajustement se fait par la méthode des moindres carrés, à partir des valeurs des O-C (différence entre les valeurs observées et les valeurs calculées). Deux drapeaux (flags) sont associés à chaque observation, un par coordonnée, ils peuvent être mis à bon ou mauvais suivant que l'observation est considérée comme bonne ou mauvaise en ascension droite ou en déclinaison. Ces drapeaux peuvent être modifiés manuellement à l'aide d'un menu déroulant, ou automatiquement à l'aide du test du $\chi^{2}$. En même temps qu'il ajuste ces paramètres, le programme fournit les écarts types sur les ajustements à effectuer. L'amélioration de l'orbite se fait donc par une succession d'intégrations et d'ajustements, et l'on arrête lorsque les améliorations des paramètres deviennent négligeables devant leurs écarts types. Le programme permet également de calculer par la méthode des moindres carrés les constantes à utiliser dans le calcul des magnitudes (magnitude du noyau et magnitude totale). 


\section{Résultats}

Après l'ajustement des éléments orbitaux, le programme permet de calculer, par intégration, de nouveaux éléments pour les époques correspondant aux passages au périhélie précédents et suivant. Ces éléments pourront être sauvegardés dans les bases de données " éléments cométaires 》 et être réutilisés par la suite pour le calcul d'éphémérides.

Les résultats, c'est-à-dire les éléments ajustés, la précision sur ces éléments, les écarts types et les moyennes des O-C, ainsi que les listes d'observations et d'O-C sont publiés et diffusés dans les Notes Orbites Cométaires $d u$ Bureau des longitudes. Le programme génère automatiquement l'édition de ces notes sur la forme de fichiers $\mathrm{T}_{\mathrm{E}} \mathrm{X}$.

Les notes sont accessibles sous la forme de fichiers Postscript sur le serveur Internet du Bureau des longitudes (ftp.bdl.fr ou www.bdl.fr), dans le répertoire $/ \mathrm{ftp} / \mathrm{pub} / \mathrm{ephem} /$ comets/elements/french pour la version française et dans le répertoire $/ \mathrm{ftp} / \mathrm{pub} / \mathrm{ephem} /$ comets/elements/english pour la version anglaise. Les éléments orbitaux calculés à l'aide de ce programme, sont utilisés pour la production des éphémérides de comètes publiées dans l'Annuaire du Bureau des longitudes et dans les Notes scientifiques et techniques du Bureau des longitudes, ils sont également utilisés dans le cadre du projet spatial ISO, pour produire les éphémérides des comètes retenues par ce projet.

\section{Références}

P. Bretagnon. (1982) Théorie du mouvement de l'ensemble des planètes. Solution VSOP82, Astron. Astrophys., Vol. no. 114, p. 278-288.

R. Bulirsh. et J. Stœr. (1980), Introduction to Numerical Analysis, Chap. no. 7, NewYork : Springer-Verlag

M. Chapront-Touzé et J. Chapront. (1983) The lunar ephemeris ELP2000, Astron. Astrophys., Vol. no. 124, p. 50-62.

G. Francou. (1983) Nouvelles éphémérides du Soleil, de la Lune et des planètes, Astron. Astrophys., Vol. no. 128, p. 124-139.

J.L. Simon. (1983) Théorie des quatre grosses planètes. Solution TOP82, Astron. Astrophys., Vol. no. 120, p. 197-202. 